\title{
Hydrodynamic interactions in dense active suspensions: from polar order to dynamical clusters
}

\author{
Natsuhiko Yoshinaga ${ }^{1,2,3, \text { f and Tanniemola B. Liverpool }}$, 4,5, † \\ 1 WPI - Advanced Institute for Materials Research, Tohoku University, Sendai 980-8577, Japan \\ 2 MathAM-OIL, AIST, Sendai 980-8577, Japan \\ 3 The Kavli Institute for Theoretical Physics, University of California, Santa Barbara, CA 93106, USA \\ ${ }^{4}$ School of Mathematics, University of Bristol, Bristol, BS8 1TW, UK \\ ${ }^{5}$ BrisSynBio, Tyndall Avenue, Bristol, BS8 1TQ, UK
}

\begin{abstract}
We study the role of hydrodynamic interactions in the collective behaviour of collections of microscopic active particles suspended in a fluid. We introduce a novel calculational framework that allows us to separate the different contributions to their collective dynamics from hydrodynamic interactions on different length scales. Hence we are able to systematically show that lubrication forces when the particles are very close to each other play as important a role as long-range hydrodynamic interactions in determining their many-body behaviour. We find that motility-induced phase separation is suppressed by near-field interactions, leading to open gel-like clusters rather than dense clusters. Interestingly, we find a globally polar ordered phase appears for neutral swimmers with no force dipole that is enhanced by near field lubrication forces in which the collision process rather than long-range interaction dominates the alignment mechanism.
\end{abstract}

PACS numbers: 87.18.Hf,64.75.Xc, 05.40.-a

Active materials are condensed matter systems selfdriven out of equilibrium by components that convert stored energy into movement. They have generated much interest recently, both as inspiration for new smart materials and as a framework to understand aspects of cell motility [1 3]. They are characterised by interesting nonequilibrium collective phenomena, such as swirling, alignment, pattern formation, dynamic cluster formation and phase separation [4 8]. Theoretical descriptions of active systems range from continuum models [1, 9] to discrete collections of self-propelled active particles [4. An influential classification of self-propelled active particle systems has been to group them into dry and wet systems [1]. Dry systems do not have momentum conserving dynamics (e.g, Vicsek models 4, 9] and Active Brownian particle (ABP) models interacting via soft repulsive potentials [10 12]), while wet systems conserve momentum via a coupling to a fluid (e.g. Squirmers driven by surface deformations [13 15] and Janus particles driven by surface chemical reactions [16]) leading to hydrodynamic interactions between active particles. Dealing with hydrodynamics leads to significant technical hurdles; as the motion of a self-propelled swimmer is affected by other particles due to both fluid flow and pressure, and even the two-body interaction between spherical squirmers in close proximity (near-field) is non-trivial, requiring sophisticated numerical analyses $[17-[23]$. Therefore, converting this into an understanding of collective behaviour remains a significant challenge [24. Because numerical simulations with hydrodynamics require significantly more computational power, studies of these systems have

\footnotetext{
*E-mail: yoshinaga@tohoku.ac.jp

${ }^{\dagger}$ E-mail: t.liverpool@bristol.ac.uk
}

relatively few particles or low resolution of fluid flow [25] 28. Hence, far-field approximations (swimmers as point multipoles ) 29 are often used to account for hydrodynamic interactions [30]. This clearly breaks down when the swimmers are close to one another, limiting the validity of such studies to very dilute suspensions.

The appearance of dynamical clusters [11, 31, 31] in recent experiments on active particles has generated much interest. This has been linked to a clustered state is observed in two-dimensional (2D) ABP systems called motility-induced phase separation (MIPS) [10 12, 32] and for squirmers confined between walls [26, 33. A major difference however is finite size clusters in experiments [7, 11, 31] compared to the infinite cluster formed in MIPS. In addition, recent simulations have shown that clusters are absent in 2D squirmer suspensions and in a 2D squirmer monolayer embedded in a 3D fluid 27, 34. While attractive interactions can lead to clustering 35] 37, here we study swimmers with purely repulsive interactions to see role of hydrodynamics in active cluster formation.

It is accepted from continuum arguments that the polar state is generically unstable for wet active systems [13], however recent simulations of wet active particles have raised the interesting possibility of other continuum limits in these systems. A polar state has been observed for neutral squirmers with no force dipole with 3D hydrodynamics, but 2D motion [19] and in 3D 38 40. It has been suggested [19] that near-field effects enhance the polar state although there are hints that far-field effects also play a role [19. These results are limited by relatively few particles so it is natural to ask if the polar state is present in the thermodynamic limit.

In this letter, we systematically construct equations of motion for wet active particles, namely, the dynamics of their position and orientation. One of our aims is to 
provide a computationally tractable model of comparable complexity to ABP which takes account of hydrodynamic interactions of particles in close proximity [17, 41]. Using it, we study a suspension of force/torque free repulsive spherical squirmers and obtain the phase behaviour summarized in Fig. 2 as a function of density $\rho_{0}$. In studying the phase behaviour, we have emphasised the dependence on the sign of the force dipole $\left(v_{2}\right)$ and contrasted them to neutral swimmers with force quadrupole and no force dipole $\left(v_{2}=0\right)$. We find significant differences between the hydrodynamic interactions with and without nearfield effects. The phase behaviour of neutral swimmers $\left(v_{2}=0\right)$ with only far-field interactions are similar to those of ABPs since there are no collision-induced reorientations. Upon including near field effects, we obtain phase diagrams characterised by at low densities, a disordered 'gas' state and at higher densities, the emergence of stable 'static clusters' except for neutral swimmers [50] which spontaneously develop polar order. Dense static clusters, present in far-field only system, are suppressed by the near-field interactions, leading to open gel-like clusters. In between the gas and static cluster are phases of 'dynamic clusters' of finite size that exchange particles with bulk. While the boundaries between different clustered phases are qualitative and threshold-dependent, the boundaries between the polar state and other states has all the features associated with a phase transition.

Each particle (squirmer) is characterized by its position and orientation $\left(\mathbf{r}^{(i)}, \mathbf{p}^{(i)}\right)$ with dynamics given by

$$
\dot{\mathbf{r}}^{(i)}=\mathbf{u}^{(i)} ; \quad \dot{\mathbf{p}}^{(i)}=\boldsymbol{\omega}^{(i)} \times \mathbf{p}^{(i)} ; \quad\left|\mathbf{p}^{(i)}\right|=1,
$$

The translational and angular velocities of each particle, $\mathbf{u}^{(i)}$ and $\boldsymbol{\omega}^{(i)}$ respectively, are obtained by solving for the

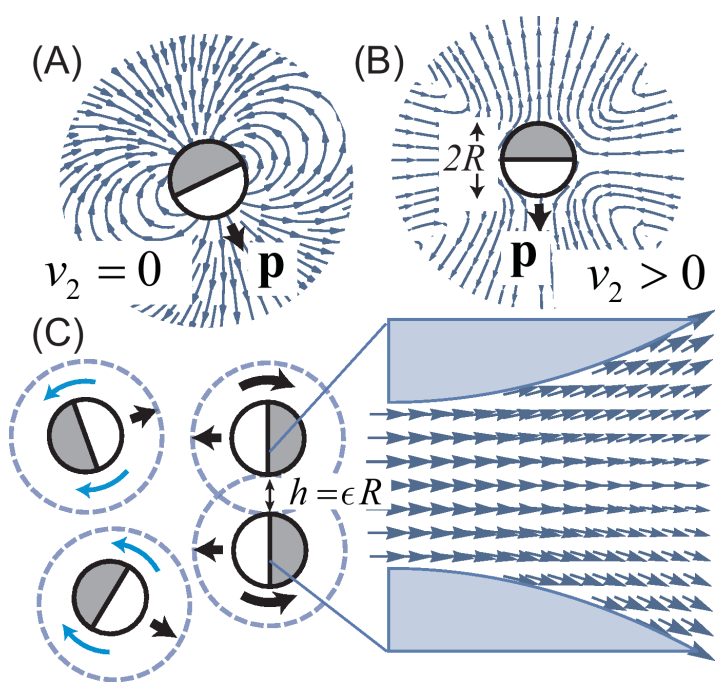

FIG. 1: (Colour Online) Schematic interaction between swimmers. Each particle creates a leading order (A) quadrupolar or (B) dipolar flow. (C) When two particles are very close to each other, lubrication flow dominates the interactions.

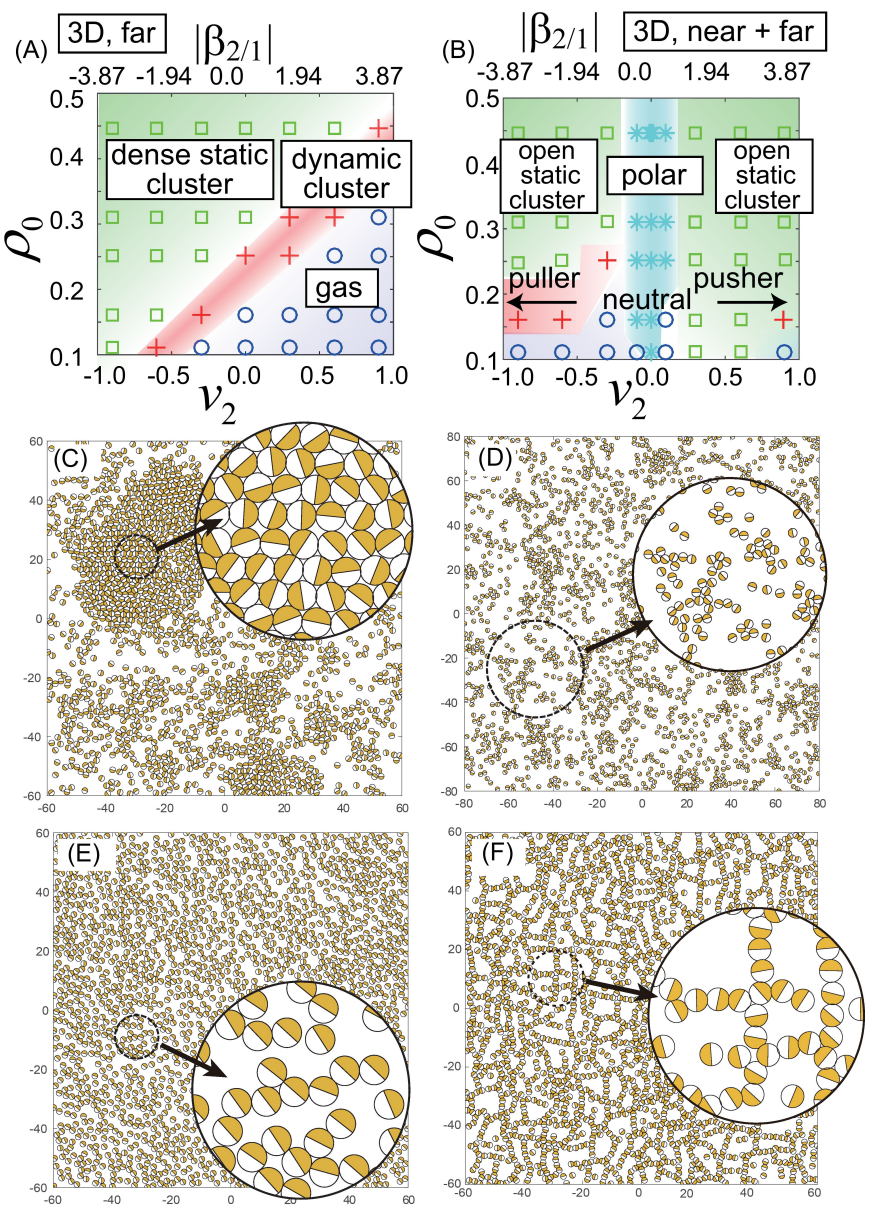

FIG. 2: (Colour Online) The state diagram of squirmers with density $\left(\rho_{0}\right)$ and dipolar strength $\left(v_{2}\right)$ with $(\mathrm{A})$ far field hydrodynamic interactions and (B) both far field and near field with $N=2048$ particles. Snap shots of (A) the static dense cluster state for a far-field-only system $\left(\phi=0.447, v_{2}=\right.$ $0)$, (B) the dynamic cluster state $\left(\phi=0.251, v_{2}=0\right)$, (C) the polar state $\left(\phi=0.447, v_{2}=0\right)$, and (D) the static open cluster state for a near+far $\operatorname{system}\left(\phi=0.447, v_{2}=0.9\right)$.

fluid mediated interaction between all pairs of particles The fluid is taken as incompressible with vanishing Re :

$$
\eta \nabla^{2} \mathbf{v}-\nabla p=0 ; \quad \nabla \cdot \mathbf{v}=0
$$

where $\eta$ is viscosity, $\mathbf{v}(\mathbf{r})$ is the velocity, and $p(\mathbf{r})$ the pressure. The boundary condition on the swimmer surface is a sum of rigid translational, $\mathbf{u}$ and rotational, $\boldsymbol{\omega}$ motion and an active slip flow, $\mathbf{v}_{s}$ driving self-propulsion:

$$
\begin{aligned}
\left.\mathbf{v}\right|_{\mathbf{r}=\mathbf{R}} & =\mathbf{u}+\boldsymbol{\omega} \times \mathbf{R}+\mathbf{v}_{s} \\
\mathbf{v}_{s} & =\sum_{l=1}^{\infty} \sum_{m=-l}^{l}\left[v_{l m} \boldsymbol{\Psi}_{l m}(\theta, \varphi)+w_{l m} \boldsymbol{\Phi}_{l m}(\theta, \varphi)\right],
\end{aligned}
$$

for a swimmer with centre at the origin. The fluid velocity vanishes at infinity, $\left.\mathbf{v}\right|_{r \rightarrow \infty}=0$, with $\theta$ the polar angle with the $z$-axis and azimuthal, $\varphi$ with the $x$-axis on 
the $x y$-plane. The slip velocity $\mathbf{v}_{s}$ can be very efficiently expanded in the tangential vector spherical harmonics, $\boldsymbol{\Psi}_{l m}$ and $\boldsymbol{\Phi}_{l m}$ 42, 43. The second term in (4) represents rotational slip around the swimmer axis associated with spinning motion which we neglect in the following and from now on set $w_{l m}=0$. The swimmer axis $\mathbf{p}$ is a unit vector (see Fig. 1). For uniaxial particles, $v_{l m}$ is a function of a magnitude $v_{l}$ and the swimmer orientation $\mathbf{p}$ [4]. An isolated squirmer moves with the velocity $\mathbf{u}_{0}^{(i)}=u_{0} \mathbf{p}^{(i)}$ with $u_{0}=-\frac{2}{3} \sqrt{\frac{3}{4 \pi}} v_{1}$.

Given two squirmers, separated by $\mathbf{r}_{i j}$, the flow field generated by one will affect the other and hence lead to modification of the self-propulsion velocities. We split the problem into two parts, a force and torque, acting on the sphere with: 1st, slip boundary conditions without translational and rotational motion $\left(\mathbf{F}^{(p)}, \mathbf{T}^{(p)}\right.$, the passive problem), and 2nd with the non-slip boundary conditions undergoing rigid-body motion $\mathbf{u}^{(i)}$ and $\boldsymbol{\omega}^{(i)}\left(\mathbf{F}^{(a)}, \mathbf{T}^{(a)}\right.$, the active problem) 43 . The force and torque-free conditions imply, $\mathbf{F}^{(a)}+\mathbf{F}^{(p)}=0$ and $\mathbf{T}^{(a)}+\mathbf{T}^{(p)}=0$ which determine $\mathbf{u}^{(i)}$ and $\boldsymbol{\omega}^{(i)}$. The problems can be solved exactly for pairs of particles in two asymptotic limits : (1) when their separation, $h_{i j}=r_{i j}-2 R, r_{i j}=\left|\mathbf{r}_{i j}\right|$ is much less than their radius (near-field) and (2) when their separation is much greater than their radius (far-field). For arbitrary separations between particles, we interpolate between the two limits; far-field and near field, using the tanh function. There is long history of calculation of the passive problem [44, 45]. Here we compute for the first time the active problem for both far-field and near-field in the general setting. Previous near-field active results have been obtained only for axisymmetric surface flowfields [17. It should be noted that to obtain the velocity and angular velocity for collections of swimmers, one must solve the active/passive problems for all possible relative orientations which has not been achieved before 23.

For a pair of squirmers (labelled $i, j$ ) with arbitrary positions (and orientation), we define a spherical coordinate system : relative separations $r_{i j}=\left|\mathbf{r}_{i j}\right|$, polar angles $\theta_{i j}$ and azimuthal angles $\varphi_{i j}$. Using it, a general form for the velocities valid in both far and near field limits is

$$
\begin{aligned}
& \mathbf{u}^{(i)}=\mathbf{u}_{0}^{(i)} \lambda^{(i)}+\sum_{\substack{j \neq i, l, m \\
\boldsymbol{\omega}^{(i)}}}=\sum_{j \neq i, l, m} \omega_{l m}^{(j i)} \mathbf{\Phi}_{l m}^{(j i)} .
\end{aligned}
$$

where $\boldsymbol{\Psi}_{l m}^{(j i)}=\boldsymbol{\Psi}_{l m}\left(\theta_{j i}, \varphi_{j i}\right), \boldsymbol{\Phi}_{l m}^{(j i)}=\boldsymbol{\Phi}_{l m}\left(\theta_{j i}, \varphi_{j i}\right)$, $\mathbf{Y}_{l m}^{(j i)}=\mathbf{Y}_{l m}\left(\theta_{j i}, \varphi_{j i}\right)$ with the $\mathbf{Y}_{l m}(\theta, \varphi)$ the normal vector spherical harmonics. For the far-field, $u_{1, m, \|}^{(j i)} \sim$ $u_{1, m, \perp}^{(j i)} \sim r_{i j}^{-3}$ (quadrupole), and $u_{2, m, \|}^{(j i)} \sim r_{i j}^{-2}$, and $\omega_{2, m}^{(j i)} \sim r_{i j}^{-3}$ (dipole). For the near-field, $u_{l m, \|}^{(j i)} \sim \epsilon \log \epsilon$ and $u_{l m, \perp}^{(j i)} \sim \omega_{l m}^{(j i)} \sim \epsilon^{0}$ with $\epsilon=\left(r_{i j}-2 R\right) / r_{i j}$ [43. $\lambda^{(i)}=1$ when the $i$ th particle is away from near-field region of any other particles and $\lambda^{(i)}=0$ otherwise.
Equations (1), 6), and (5) form a closed complete dynamical system. Using them, we performed numerical simulations of $N$ identical particles of radius $R$ with periodic boundary conditions. Figure 2 shows various state points of this model as a function of the density, $\rho_{0}=\pi R^{2} N / L^{2}$ and the force dipole strength $v_{2}$. Most have $N=2048$ unless specified otherwise. Defining, the average distance between two particles, $\xi=R \sqrt{\pi / \rho_{0}}$, we vary $\xi$ from $\xi \simeq 2.65$ to $\xi \simeq 5.30$. We set $v_{1}=1$ for all swimmers and thus $u_{0} \simeq 0.32$.

The size of a particle is chosen to be of unit length, thus we set $R=1$. The time scale is normalised by the time for an isolated squirmer to move a half of its body length, that is, $\tau_{0}=R / u_{0}$. There is a time scale associated with collisions, $\tau_{m}=\xi / u_{0}$. We vary the time scale from $\tau_{m} \simeq 8$ to $\tau_{m} \simeq 17$. We consider motion restricted to $2 \mathrm{D}$ but interacting via $3 \mathrm{D}$ hydrodynamic interactions. We neglect the modes with $l \geq 3$. We note that for 3D hydrodynamics projected onto $2 \mathrm{D}$, pushers and pullers are not identical; the interaction at the front and the back is stronger than that at the side. As a result, pullers, on average attract nearby objects. We find global phase separation of active particles with repulsive interactions, i.e. MIPS, is suppressed by near-field hydrodynamics and we find instead networks of open clusters for a large range of intermediate densities. We see a gel-like extended state at high enough densities.

Most surprisingly we find that for neutral (quadrupolar) squirmers and squirmers with small dipoles, $\left|v_{2}\right| \ll 1$, the swimmers self-organise into a polar state with aligned orientations and swimming directions. This polar order vanishes at low density. Screening far-field interactions [46] leads to polar order at lower densities (see Fig. 3(C)). As $\left|v_{2}\right|$ is increased, polar order vanishes. For example, for pushers $\left(v_{2}>0\right)$, polar order disappears at $v_{2}^{*} \simeq 0.15\left(\beta_{2 / 1} \simeq 0.58\right)$ in Fig. 3(A). The loss of polar order is accompanied by divergence of fluctuations of the polarity as shown in Fig. 3(A). The position of the phase boundary is not symmetric about $v_{2}=0$, i.e. different $\left|v_{2}^{*}\right|$ for pushers and pullers (see Fig. $3(\mathrm{~B})$ ).

We check the stability of polar order to fluctuations by adding Gaussian white noise of amplitude $\sigma$ to the rotation in eqn. (1). At a fixed density, $\rho_{0}$ we find a transition from a gas to a polar state at a critical value of $\sigma_{c}>0$. Polar order remains as we increase system size. In Fig. 3(D), polar order is shown as a function of the number of particles, $N$ up to $N=8192$ for the near+far field system and $N=32768$ for the near-field-only system. Therefore we conclude that the system is truly in a state with macroscopic global polar order. These system sizes are comparable to ABPs and the Vicsek models. The mean cluster ratio, $\langle q\rangle$, the fraction of swimmers in large clusters, is nearly zero throughout the polar phase (see Fig. 3(D)), indicating clusters are not associated with polar order.

It is evident from the simulations that collisions between the particles are key in the development of polar order. Hence, we explore a two body collision in detail 

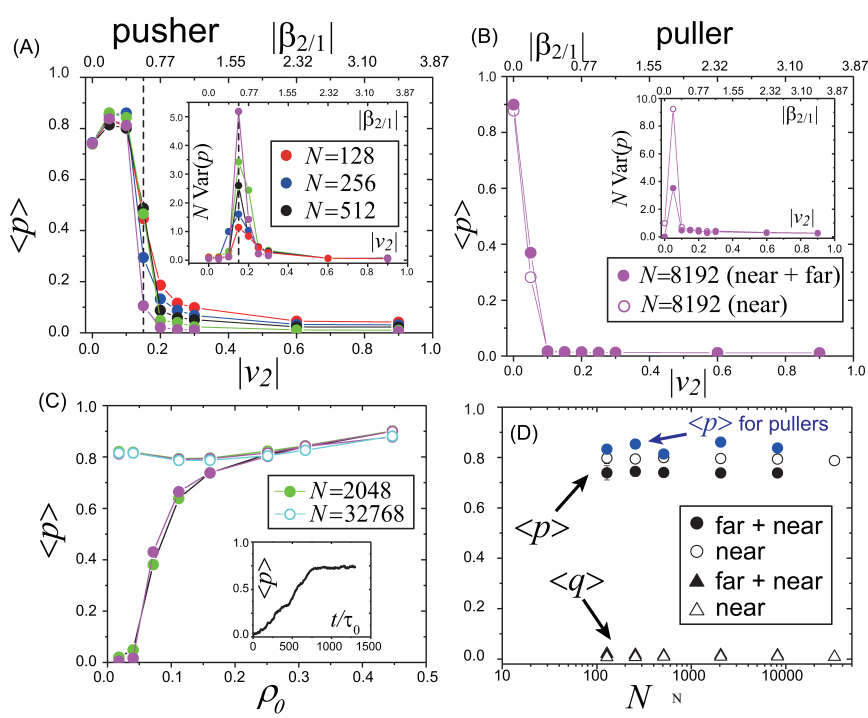

FIG. 3: (Colour Online) (A, B) The mean polarity as a function of the dipolar slip flow $v_{2}$ on a squirmer at $\rho_{0}=0.16$ $(\xi=4.43)$. The corresponding values of the squirmer index $\beta_{2 / 1}$ is shown at the upper axis. The polar order $\langle p\rangle$ for pushers (A) and pullers (B). The insets in (A) and (B) show the variance of the polar order. Closed circles indicate the system with the near and far fields whereas open circles indicate the near-field-only system. (C) the mean polarity, $\langle p\rangle$ for neutral swimmers $v_{2}=0$ vs density, $\rho_{0}$ (inset: time evolution of $\langle p\rangle$ ). The solid (open) symbols correspond to the simulations with (without) the far-field interaction. Both include the near field interaction. (D) The system size dependence of the mean polar order $\langle p\rangle$ and the mean cluster ratio $\langle q\rangle$ at $\rho_{0}=0.16(\xi=4.43)$ of the neutral swimmer $v_{2}=0$ (black). Both the simulations with far- and near-field interactions and only near-field interactions are shown. The results of the simulations with far- and near-field interactions for weak pushers $v_{2}=0.05$ are also shown in blue. The legends are shared by all the figures.

(see Fig. 4(A) and 43]). Figure 4(B) shows some trajectories of two 'colliding' squirmers.

For the far-field only system, any transient alignment of pairs of squirmers is unstable to rotational fluctuations arising from collisions with other particles and no polar order is developed. Including the near-field (lubrication) interaction however leads to reorientation while in transient bound (Fig. 4(A)) states occurring during collisions as shown in Fig. 4(D). For small incident angles $\left(\left|\phi_{0}\right| \lesssim \pi / 4\right)$, collisions are symmetric, i.e the reflection angle $\left(\phi_{f}\right)$ equals $\phi_{0}$ but for as $\left|\phi_{0}\right|$ increases, $\left|\phi_{f}\right|$ stops increasing and tends to a finite (saturation) angle $\lesssim \pi / 4$. This asymmetry between incident and reflection angles means $\left(\left\langle\left|\phi_{f}\right|\right\rangle\left\langle\left\langle\left|\phi_{0}\right|\right\rangle\right.\right.$, see Fig. 4(C)) and leads eventually to alignment. This effect is most pronounced for neutral swimmers $\left(v_{2}=0\right)$; while similar behaviour is seen for pushers and pullers, shorter residence times for pullers (Fig. 4(D)) and larger reflection angles for pushers (Fig. 4.C)) eventually destroy the polar state for both

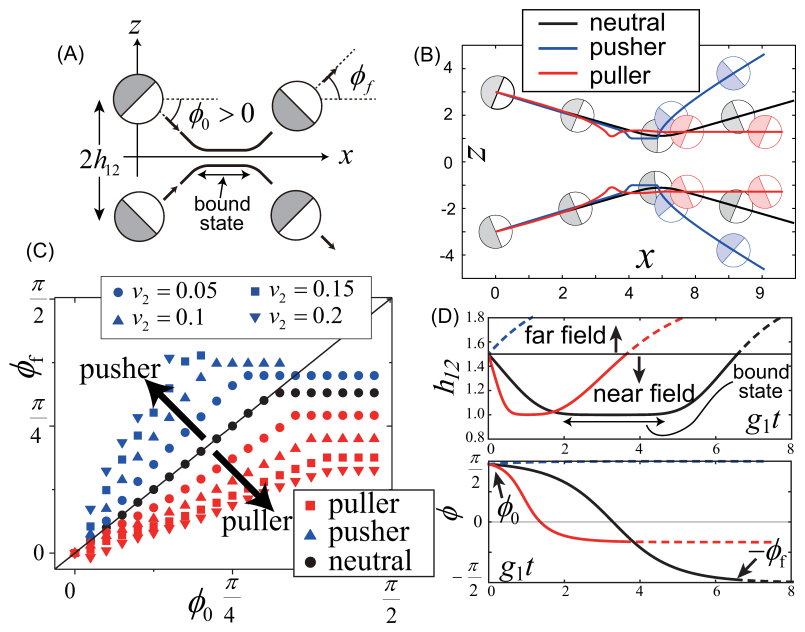

FIG. 4: (Colour Online) (A) Schematics of two-body collisions. (B) Typical trajectories for neutral, pusher, and puller swimmers are shown. (C) The incidence $\phi_{0}$ and reflection $\phi_{f}$ angles for symmetric collisions for neutral swimmers $\left(v_{2}=0\right)$ and small deviation of $v_{2}$ to pushers $\left(v_{2}>0\right)$ and pullers $\left(v_{2}<0\right)$. The solid line shows $\phi_{0}=\phi_{f}$. Only the range of $0 \leq \phi_{0}, \phi_{f} \leq \pi / 2$ is shown. (D) The dynamics of the separation $h_{12}(t)$ and the angle $\phi(t)$ in the near-field region for $g_{2} / g_{1}=1$. Here, $g_{1} \sim u_{0} / R$ and $g_{2} \sim v_{2} / R[43$. The time scale is normalised by $g_{1}^{-1}$. Motion outside the near-field region is indicated by dashed lines. The legends are shared by all the figures.

of them as $\left|v_{2}\right|$ becomes large. The saturation angle in Fig. 4. C) is due to direct contacts between squirmers (repulsive forces from the interaction potential). This reorientation depends weakly on the contact interaction; big changes of interaction potential lead only to slight shifts of saturation angles. Hence the collective behaviour and phase boundaries are independent of the choice of potential [43.

While we only considered pairwise interactions, combining many of them results in many-body effects which become relevant for a non-dilute suspension. In fact, for a dense suspension, dynamics is dominated by the lubrication interaction between swimmers which is well approximated by a sum of two-body interactions. In particular, $\langle p\rangle$ is independent of $N$ even for weak pushers $v_{2} \neq 0$ as shown in Fig. 3(D). To understand how these many-body effects give rise to collective behaviour, we have carried out numerical simulations of a minimal model, in which the only non-zero interactions are rotational near-field: $\omega_{l m}^{(i j)} \sim \epsilon^{0} \quad 43$ plus noise. It has two key ingredients : short-range orientational interactions and short-range repulsive interactions. We are able to reproduce the same polar-disorder phase transition by increasing the noise amplitude. We conclude that the detailed form of the hydrodynamic interactions are not essential for the development of polar order. In contrast to the Vicsek model, here the lack of an alignment rule means excluded volume interactions are required to generate polar order. 
The existence of polar order is fundamentally surprising because of the apparent contradiction with the well accepted generic instability of polar/nematic order of wet active matter [1, 47]. To understand this we construct a two-fluid model for the system: the suspending fluid (volume fraction, $1-\phi)$ with velocity $\mathbf{v}(\mathbf{r}, t)$ and the active particle (squirmer) 'phase' (volume fraction $\phi$ ) with local displacement variable $\mathbf{u}(\mathbf{r}, t)$ due to squirmer density variations. Finally we identify a local polar order parameter, $\mathbf{p}(\mathbf{r}, t)$. Our analysis highlights collisions of the swimmers as essential for the formation of polar order. An isolated squirmer swims with velocity $u_{0} \mathbf{p}$ relative to the background fluid. The fluid obeys the Stokes equation with a force density $\mathbf{f}^{c} \sim O\left(\rho_{a}^{2}\right)$ due to collisions between squirmers and an active stress $\sigma_{i j}^{a}=\zeta p_{i} p_{j}$ where $\zeta \propto v_{2}$ and $\rho_{a}=\phi \rho$ is the average density of active particles. Replacing $\mathbf{f}^{c}$ by $\chi\left(\dot{\mathbf{u}}-\mathbf{v}-u_{0} \mathbf{p}\right)$. and linearising $\mathbf{p}, \mathbf{v}$ about the homogeneous state: $\mathbf{v}_{0}=\dot{\mathbf{u}}_{0}-u_{0} \mathbf{p}_{0}$,

$$
\begin{aligned}
0 & =\eta \nabla^{2} \delta \mathbf{v}+\chi \delta \mathbf{v}-\nabla P+\nabla \cdot \boldsymbol{\sigma}^{a} ; \nabla \cdot \delta \mathbf{v}=0,(7) \\
\partial_{t} \delta \mathbf{p} & =-u_{0} \mathbf{p}_{0} \cdot \nabla \delta \mathbf{p}+\delta \boldsymbol{\omega} \cdot \mathbf{p}_{0}+\gamma \delta \mathbf{e} \cdot \mathbf{p}_{0}+K \nabla^{2} \delta \mathbf{p}(8)
\end{aligned}
$$

where $\omega_{i j}=\frac{1}{2}\left(\partial_{i} v_{j}-\partial_{j} v_{i}\right), e_{i j}=\frac{1}{2}\left(\partial_{i} v_{j}+\partial_{j} v_{i}\right)$ and $K$ is the Frank elastic constant in the one constant approximation. A finite screening length $\xi \sim \sqrt{\eta / \chi}$ weakens the generic instability from long-wavelengths to finite wavelengths and stabilises the polar state on long lengthscales. Hence a comparison between the screening length $\xi$ and the active lengthscale, $\sqrt{K /|\zeta|}[48$, allows us to determine the onset of polar order for $|\zeta|<\zeta_{c}=K / \xi^{2}$, i.e. for swimmers that are close to neutral. We recover the generic instability as $\phi \rightarrow 0$.

We emphasise that the computational expense of including hydrodynamics in the simulations of collective behaviour of active matter requires trade-offs where accuracy is sacrificed. Navier-Stokes (NS) solvers such as Lattice-Boltzmann compromise on the resolution of the velocity field and hence do not accurately describe fluid flow when the active particles are very close to each other. Here we have developed another scheme whose strengths are exactly where NS solvers are weak, for active particles in close proximity. It is also very accurate when the particles are well separated. Where it is less accurate, however is at intermediate separations. Its other great advantage is the ease with which we can study systems with many more particles. Another nice feature is the ability to switch off different contributions to the motion to identify the dominant mechanisms behind the macroscopic phenomena observed. Using it we have studied the collective behaviour of large numbers of spherical active particles and confirmed and clarified the phenomena observed in smaller simulations. Dense cluster formation is suppressed and we show that it is replaced by open gellike aggregates at higher densities and most surprisingly, a polar ordered phase is stabilised by hydrodynamic lubrication interactions. We have also provided analytic continuum arguments explaining how such a state can be realised. In addition to the work presented here, we have studied purely $2 \mathrm{D}$ systems (2D with $2 \mathrm{D}$ interactions) and $3 \mathrm{D}$ systems (3D with $3 \mathrm{D}$ interactions), and obtained similar results, but at higher densities 49 .

\section{Acknowledgments}

The authors are grateful to S. Fielding, T. Ishikawa and R. Golestanian for helpful discussions. NY acknowledges the support by JSPS KAKENHI grant numbers JP26800219, JP26103503, and JP16H00793. NY also acknowledges the support by JSPS A3 Foresight Program. TBL is supported by BrisSynBio, a BBSRC/EPSRC Advanced Synthetic Biology Research Center (grant number BB/L01386X/1). We would like to thank the Isaac Newton Institute for Mathematical Sciences, Cambridge, for support and hospitality during the programmes, "The Mathematics of Liquid Crystals" and "Dynamics of active suspensions, gels, cells and tissues" where work on this article was started.
[1] M. C. Marchetti, J. F. Joanny, S. Ramaswamy, T. B. Liverpool, J. Prost, Madan Rao, and R. Aditi Simha. Hydrodynamics of soft active matter. Rev. Mod. Phys., 85:1143-1189, 2013.

[2] J. Toner, Y. Tu, and S. Ramaswamy. Hydrodynamics and phases of flocks. Ann. Phys., 318(1):170-244, 2005.

[3] Sriram Ramaswamy. The mechanics and statistics of active matter. Ann. Rev. Cond. Mat. Phys., 1:323-345, 2010.

[4] Tamás Vicsek, András Czirók, Eshel Ben-Jacob, Inon Cohen, and Ofer Shochet. Novel type of phase transition in a system of self-driven particles. Phys. Rev. Lett., 75(6):1226-1229, Aug 1995.

[5] Henricus H. Wensink, Jörn Dunkel, Sebastian Heidenreich, Knut Drescher, Raymond E. Goldstein, Hartmut Löwen, and Julia M. Yeomans. Meso-scale turbulence in living fluids. Proc. Nat. Acad. Sci., 109(36):14308-14313, 2012.

[6] M E Cates. Diffusive transport without detailed balance in motile bacteria: does microbiology need statistical physics? Rep. Prog. Phys., 75(4):042601, 2012.

[7] Jeremie Palacci, Stefano Sacanna, Asher Preska Steinberg, David J. Pine, and Paul M. Chaikin. Living crystals of light-activated colloidal surfers. Science, 339(6122):936-940, 2013.

[8] Antoine Bricard, Jean-Baptiste Caussin, Nicolas Desreumaux, Olivier Dauchot, and Denis Bartolo. Emergence of macroscopic directed motion in populations of motile colloids. Nature, 503(7474):95-98, 2013.

[9] Eric Bertin, Hugues Chaté, Francesco Ginelli, Shradha Mishra, Anton Peshkov, and Sriram Ramaswamy. Meso- 
scopic theory for fluctuating active nematics. New Journal of Physics, 15(8):085032, 2013.

[10] Yaouen Fily and M. Cristina Marchetti. Athermal phase separation of self-propelled particles with no alignment. Phys. Rev. Lett., 108:235702, Jun 2012.

[11] Ivo Buttinoni, Julian Bialké, Felix Kümmel, Hartmut Löwen, Clemens Bechinger, and Thomas Speck. Dynamical clustering and phase separation in suspensions of self-propelled colloidal particles. Phys. Rev. Lett., 110:238301, Jun 2013.

[12] Gabriel S. Redner, Michael F. Hagan, and Aparna Baskaran. Structure and dynamics of a phase-separating active colloidal fluid. Phys. Rev. Lett., 110:055701, 2013.

[13] M. J. Lighthill. On the squirming motion of nearly spherical deformable bodies through liquids at very small reynolds numbers. Communications on Pure and Applied Mathematics, 5(2):109-118, 1952.

[14] JR Blake. Self propulsion due to oscillations on the surface of a cylinder at low reynolds number. Bulletin of the Australian Mathematical Society, 5(02):255-264, 1971.

[15] OnShun Pak and Eric Lauga. Generalized squirming motion of a sphere. Journal of Engineering Mathematics, 88(1):1-28, 2014.

[16] Ramin Golestanian, Tanniemola B. Liverpool, and Armand Ajdari. Propulsion of a molecular machine by asymmetric distribution of reaction products. Physical Review Letters, 94(22):220801, 2005.

[17] T. Ishikawa, MP Simmonds, and TJ Pedley. Hydrodynamic interaction of two swimming model microorganisms. J. Fluid Mech., 568:119-160, 2006.

[18] I. Llopis and I. Pagonabarraga. Dynamic regimes of hydrodynamically coupled self-propelling particles. Eur. Phys. Lett., 75(6):999, 2006.

[19] Takuji Ishikawa, J. T. Locsei, and T. J. Pedley. Development of coherent structures in concentrated suspensions of swimming model micro-organisms. J. Fluid Mech., 615(-1):401-431, 2008.

[20] Kenta Ishimoto and Eamonn A. Gaffney. Squirmer dynamics near a boundary. Phys. Rev. E, 88:062702, Dec 2013.

[21] Gao-Jin Li and Arezoo M. Ardekani. Hydrodynamic interaction of microswimmers near a wall. Phys. Rev. E, 90:013010, Jul 2014.

[22] Nima Sharifi-Mood, Ali Mozaffari, and Ubaldo CórdovaFigueroa. Pair interaction of catalytically active colloids: From assembly to escape. arXiv:1510.03000 2015.

[23] Dario Papavassiliou and Gareth P. Alexander. Exact solutions for hydrodynamic interactions of two squirming spheres. arXiv:1602.06912, 2016.

[24] Peter J. Mucha, Shang-You Tee, David A. Weitz, Boris I. Shraiman, and Michael P. Brenner. A model for velocity fluctuations in sedimentation. Journal of Fluid Mechanics, 501:71-104, 022004.

[25] John J. Molina, Yasuya Nakayama, and Ryoichi Yamamoto. Hydrodynamic interactions of self-propelled swimmers. Soft Matter, 9:4923-4936, 2013.

[26] Andreas Zöttl and Holger Stark. Hydrodynamics determines collective motion and phase behavior of active colloids in quasi-two-dimensional confinement. Phys. Rev. Lett., 112:118101, Mar 2014.

[27] Ricard Matas-Navarro, Ramin Golestanian, Tanniemola B. Liverpool, and Suzanne M. Fielding. Hydrodynamic suppression of phase separation in active suspensions. Phys. Rev. E, 90:032304, Sep 2014.
[28] J.-B. Delfau, J. Molina, and M. Sano. Collective behavior of strongly confined suspensions of squirmers. Eur. Phys. Lett., 114(2):24001, 2016.

[29] Saverio E Spagnolie and Eric Lauga. Hydrodynamics of self-propulsion near a boundary: predictions and accuracy of far-field approximations. Journal of Fluid Mechanics, 700:105-147, 2012.

[30] D. Saintillan and M. J. Shelley. Complex Fluids in Biological Systems, chapter Theory of active suspensions, pages 319-355. Springer, 2015.

[31] I. Theurkauff, C. Cottin-Bizonne, J. Palacci, C. Ybert, and L. Bocquet. Dynamic clustering in active colloidal suspensions with chemical signaling. Phys. Rev. Lett., 108:268303, Jun 2012.

[32] Fernando Peruani, Andreas Deutsch, and Markus Bär. Nonequilibrium clustering of self-propelled rods. Phys. Rev. E, 74(3):030904, Sep 2006.

[33] Johannes Blaschke, Maurice Maurer, Karthik Menon, Andreas Zöttl, and Holger Stark. Phase separation and coexistence of hydrodynamically interacting microswimmers. Soft Matter, 12:9821-9831, 2016.

[34] P G Saffman and M Delbrck. Brownian motion in biological membranes. Proc. Nat. Acad. Sci., 72(8):3111-3113, 1975.

[35] Suropriya Saha, Ramin Golestanian, and Sriram Ramaswamy. Clusters, asters, and collective oscillations in chemotactic colloids. Phys. Rev. E, 89:062316, Jun 2014.

[36] Ricard Matas Navarro and Suzanne M. Fielding. Clustering and phase behaviour of attractive active particles with hydrodynamics. Soft Matter, 11:7525-7546, 2015.

[37] Francisco Alarcon, Chantal Valeriani, and Ignacio Pagonabarraga. Morphology of clusters of attractive dry and wet self-propelled spherical particle suspensions. Soft Matter, pages -, 2016.

[38] Arthur A. Evans, Takuji Ishikawa, Takami Yamaguchi, and Eric Lauga. Orientational order in concentrated suspensions of spherical microswimmers. Physics of Fluids, 23(11):-, 2011.

[39] F. Alarcón and I. Pagonabarraga. Spontaneous aggregation and global polar ordering in squirmer suspensions. Journal of Molecular Liquids, 185:56 - 61, 2013. Molecular Simulations of Complex Systems.

[40] John Jairo Molina Norihiro Oyama and Ryoichi Yamamoto. A binary collision route for purely hydrodynamic orientational ordering of microswimmers. arXiv:1606.03839, 2016.

[41] James W. Swan, John F. Brady, Rachel S. Moore, and ChE 174. Modeling hydrodynamic self-propulsion with stokesian dynamics. or teaching stokesian dynamics to swim. Physics of Fluids, 23(7), 2011.

[42] E. L. Hill. The theory of vector spherical harmonics. Am. J. Phys., 22(4):211-214, 1954.

[43] See Supplemental Material at http://link.aps.org/supplemental/???.?????? for details of the fomula and the numerical simulations.

[44] DJ Jeffrey and Y Onishi. Calculation of the resistance and mobility functions for two unequal rigid spheres in low-reynolds-number flow. Journal of Fluid Mechanics, 139:261-290, 1984.

[45] S. Kim and S.J. Karrila. Microhydrodynamics. Butterworth-Heinemann New York, 1991.

[46] R.C. Ball and J.R. Melrose. A simulation technique for many spheres in quasi-static motion under frameinvariant pair drag and brownian forces. Physica A, 
247(1-4):444 - 472, 1997.

[47] R. Aditi Simha and Sriram Ramaswamy. Hydrodynamic fluctuations and instabilities in ordered suspensions of self-propelled particles. Phys. Rev. Lett., 89(5):058101, Jul 2002.

[48] R Voituriez, JF Joanny, and J Prost. Spontaneous flow transition in active polar gels. EPL (Europhysics Letters), 70(3):404, 2005.

[49] N. Yoshinaga and T. B. Liverpool (unpublished).

[50] See 43 for the definitions of the polar order and the clusters 